\title{
A study of trailing-edge losses in Organic Rankine Cycle turbines
}

\author{
Francisco J. Durá Galiana* \\ Aerodynamics and Flight Mechanics \\ Engineering and the Environment \\ University of Southampton \\ Southampton, UK \\ Email: fjdg1g08@soton.ac.uk
}

\author{
Andrew P.S. Wheeler \\ Whittle laboratory \\ Engineering Department \\ University of Cambridge \\ Cambridge, UK
}

\author{
Jonathan Ong \\ GE Global Research \\ Munich, Germany
}

In this paper vane trailing-edge losses which occur in Organic Rankine Cycle (ORC) turbines are investigated. Experiments are performed to study the influence of dense gas effects on trailing-edge loss in supersonic flows using a novel Ludwieg tube facility for the study of dense-gas flows. The data is also used to validate a CFD flow solver. The computational simulations are then used to determine the contributions to loss from shocks and viscous effects which occur at the vane trailing-edge. The results show that dense gas effects play a vital role in the structure of the trailing-edge flow, and control the extent of shock and viscous losses.

\section{Nomenclature}

a Speed of sound

$h$ Enthalpy

$h_{s} \quad$ Isentropic enthalpy

$h_{\circ} \quad$ Stagnation enthalpy

$M$ Mach number

$s$ Entropy

$T$ Temperature

$T_{c} \quad$ Temperature at critical point

$T_{S} \quad$ Isentropic temperature

$T_{\circ} \quad$ Stagnation temperature

$t_{\text {t.e. }} \quad$ Trailing-edge thickness

$t$ Time

$p$ Pressure

$p_{c} \quad$ Pressure at critical point

$p_{\circ} \quad$ Stagnation pressure

$p_{b}$ Base pressure

$v$ Specific volume

${ }^{*}$ Corresponding author.

\section{GREEK LETTERS}

$\Gamma$ Fundamental derivative

$\theta_{w} \quad$ Trailing-edge separation angle

$\rho$ Density

$\checkmark$ Prandtl-Meyer function

$\zeta$ Loss coefficient

\section{INTRODUCTION}

Dense gases are commonly used in Organic Rankine $\mathrm{Cy}$ cle (ORC) engines, which are employed in the field of heat recovery in industrial processes and power generation. ORC engines are particularly effective at producing power from low temperature heat sources and often have a single stage radial turbine to increase the power density. This helps reduce costs but generates high pressure ratios across the turbine. When the turbine pressure ratio is high, transonic flows and high losses related to supersonic effects can occur. In addition to shock losses, the expansion in such turbines occurs close to the saturation line of the gas where dense gas effects can be significant.

Dense gas effects are present in flows in which the Fundamental derivative [1] has a value below unity. This is generally achieved by gases with high molecular weights and complexity [2]. The Fundamental derivative is given by:

$$
\Gamma=\frac{a^{4}}{2 v^{3}}\left(\frac{\partial^{3} v}{\partial p^{2}}\right)=1+\frac{\rho}{a}\left(\frac{\partial a}{\partial \rho}\right)_{s}
$$

For ordinary gases, such as steam, the value of the Fundamental derivative, $\Gamma$, is greater than 1 , meaning that the speed of sound decreases through an expansion. The rate of change of the speed of sound with pressure decreases as the 
Fundamental derivative is reduced. When $\Gamma<1$ the speed of sound increases throughout an expansion. Another effect seen when reducing the Fundamental derivative is that the value of the Prandtl-Meyer function, $v$, is increased, for a given Mach number [3]. This increase in the Prandtl-Meyer function means that, in order to accelerate a sonic flow to a given supersonic Mach number, a gas with a lower Fundamental derivative requires a higher turning angle or area ratio.

At certain conditions some thermodynamic models show the gas can enter a region of non-classical behaviour delimited by $J=0$ [4], where

$$
J=-\frac{v}{M}\left(\frac{\mathrm{d} M}{\mathrm{~d} v}\right)=1-\Gamma-\frac{1}{M^{2}}
$$

In this region (where $J>0$ ) classical gas dynamics are inverted; expansion waves converge forming expansion shocks and compression waves form compression fans. To the author's knowledge there has been only one experimental investigation showing non-classical effects [5], but computational simulations have shown these effects may exist in ORC turbines [6-13].

The study of dense gases in turbines is an emerging field of study. A few studies have looked at viscous effects in dense gas flows (for instance [14-18]); these studies have tended to show reduced viscous effects within the dense-gas region, although it is unclear whether the changes in viscous effects in these studies were due to the high Reynolds numbers which occur at dense gas conditions.

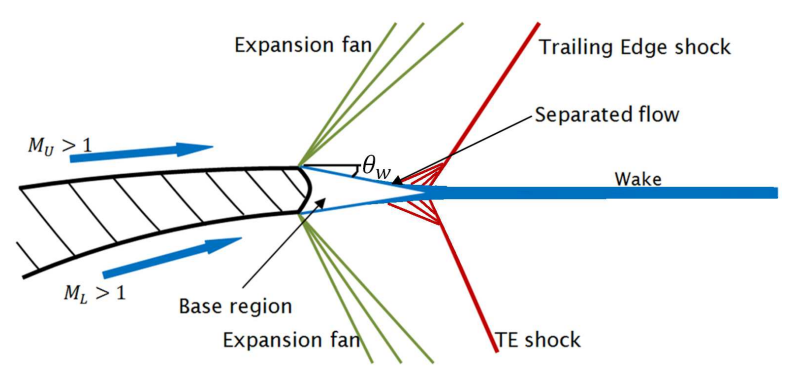

Fig. 1. FLOW STRUCTURE AROUND A TRAILING EDGE IN A SUPERSONIC FLOW

The present investigation focuses on the vane trailingedge flow within an ORC turbine, since this is a region where strong dense gas effects are likely to occur [3,19]. Within a typical turbine, the trailing-edge flow is strongly dependent on Mach number and can account for a third of the total stage losses [20], which makes the trailing-edge region critical to turbine efficiency, particularly for transonic turbines. Generally for a vane with supersonic exit flow, the trailing-edge flow structure is composed of: an expansion-shock system; flow separation; a base flow region; and a free shear wake (Fig. 1). The expansion fans on either side of the trailingedge are generated as the flow accelerates around the sharp
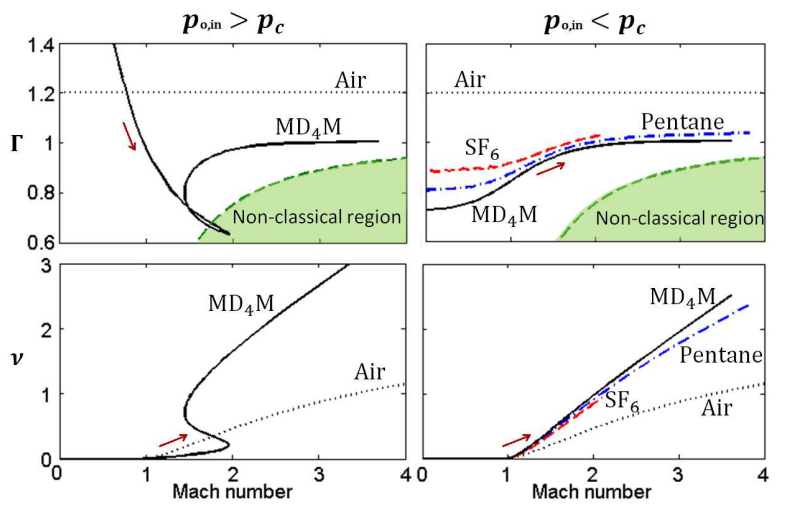

Fig. 2. CHANGE IN FUNDAMENTAL DERIVATIVE, $\Gamma$, AND PRANDTL-MEYER FUNCTION, $v$, ALONG SUPERCRITICAL $p_{o}=$ $2 p_{c}, T_{o}=1.025 T_{c}(\mathrm{LEFT})$ AND SUBCRITICAL $p_{o}=0.9 p_{c}, T_{o}=$ $1.01 T_{c}$ (RIGHT) EXPANSIONS FOR SEVERAL GASES

trailing-edge corner; this produces a region of separation followed by a confluence region, where shock-waves are produced as the flows from either side of the blade/vane meet and turn in a shared direction. As a result of all this both viscous and inviscid mechanisms work together producing loss through intense mixing and shock-loss.

Up to now there have been no studies specifically focussing on the trailing-edge region within ORC turbines, although the requirement for higher pressure ratio single-stage turbines, and thus higher vane exit Mach numbers, will mean that trailing-edge effects will become increasingly important to ORC turbine designs. Furthermore, since the trailing-edge flow-field is strongly dependent on the coupling between the inviscid flow field (controlled by the flow gas dynamics) and the viscous flow field, the influence of dense-gas effects are likely to play an important role in this region.

This paper presents an experimental and computational study of ORC turbine vane trailing-edge flows. The first part of the paper discusses how the gas-dynamics of various working-fluids changes when operating within the dense-gas region; this involves examining the variations in PrandtlMeyer function over a wide range of conditions for various fluids. Secondly, an experimental study on trailing-edge wake flows is discussed. The results are then compared with CFD predictions using both RANS and LES modelling. Finally, CFD simulations of an ORC vane are performed in order to determine the contributions to loss from the viscous and inviscid flow fields.

\section{EXPANSION OF DENSE GAS FLOWS}

To assess the behaviour of the inviscid dense gas dynamics in typical ORC turbines, a numerical study was performed using the National Institute of Standards and Technology (NIST) real gas routines (REFPROP) [21]. The equations of state used by REFPROP can be found in $[22,23]$ and are based on a Helmholtz functional form. The following results are purely determined from gas phase data and twophase regions are not considered. 
The aim of the study was to determine how the gas properties of several working fluids would change across an isentropic turbine vane operating over a range of inlet stagnation conditions. This enables an initial estimate of how the gas dynamics of different working fluids are expected to change within a turbine vane.

In this study the properties of several gases were determined along isentropic changes in pressure. By setting the initial stagnation conditions $\left(p_{o}, T_{o}\right)$, expansions were produced from super-critical stagnation pressures and subcritical stagnation pressures to low values of pressure ( $p=$ $\left.0.001 p_{c}\right)$. Stagnation temperatures were set so that the flow passed through the dense gas region. Along each isentropic expansion, the values of Mach number, Prandtl-Meyer function $v, J$ and $\Gamma$ were determined.

Figure 2 shows example results from this study. The plot on the top left shows the variation of fundamental derivative with Mach number for a siloxane $(M D 4 M)$ at a supercritical inlet stagnation condition of $p_{o}=2.0 p_{c}, T_{o}=1.025 T_{c}$ and the arrow indicates the direction of reducing pressure. For reference, the fundamental derivative for Air is also shown, assuming air operates as an ideal gas and as such $\Gamma$ is constant. As $M D 4 M$ is expanded from rest up to $M=2$, there is a large reduction in fundamental derivative $\Gamma$. The reduction in fundamental derivative is large enough that the gas enters the non-classical region, where $J=1-\Gamma-\frac{1}{M^{2}}>0$; this means that further expansion of the gas leads to a reduction in Mach number, and there is a subsequent increase in $\Gamma$ such that the gas re-enters the classical region. Thus, within the non-classical region, there is a local maximum possible Mach number at which the fundamental derivative is a minimum, and $\mathrm{J}$ is a maximum. As the gas continues to expand, the Mach number continues to fall until a local minimum in Mach number is reached, after which the Mach number (and $\Gamma)$ then continuously rises as pressure reduces. Below this plot is shown the variation in Prandtl-Meyer function $v$ with Mach number at these same conditions. The plot shows that the local maximum in Mach number within the non-classical region, and local minimum in Mach number as the flow expand out of the non-classical region are both regions where the rate of change of $v$ with Mach number becomes infinite; this means that very large changes in flow direction or flow area will have very little effect on Mach number in these regions, and thus in practise it may be very difficult to design a nozzle to achieve these conditions.

In order to determine the likely extent of non-classical behaviour within ORC turbines, the maximum value of $J$ was determined for various fluids over a broad range of conditions, and the value of turbine inlet stagnation conditions required to achieve this condition determined using the method described above assuming an isentropic expansion in the turbine. The results are shown in Tab. 1, which shows that the turbine inlet conditions at which $J$ is maximized are generally a high supercritical pressure $\left(p_{o} / p_{c} \approx 4\right)$ and a supercritical temperature which is in the range $1<T_{o} / T_{c}<1.6$. Significant regions of non-classical flow were only observed for the siloxanes $(M D M, M D 4 M)$ over the range of conditions studied, and again these conditions were only observed at high supercritical pressures and temperatures close to $T_{c}$.

Table 1. MAXIMUM J FOR VARIOUS FLUID EXPANSIONS FROM THE RANGE OF INITIAL CONDITIONS $0.5<p_{o} / p_{c}<3.7$ AND $0.7<T_{o} / T_{c}<1.6$ WHERE $p_{\min }=0.001 p_{c}$

\begin{tabular}{|c|c|c|c|}
\hline Fluid & $J_{\max }$ & $p_{\circ} / p_{c}$ & $T_{\circ} / T_{c}$ \\
\hline$M D 4 M$ & 0.2574 & 3.66 & 1.03 \\
$M D M$ & 0.2324 & 3.66 & 1.045 \\
Pentane & 0.0138 & 3.58 & 1.105 \\
$R 218$ & -0.0263 & 3.66 & 1.12 \\
$R 245 f a$ & -0.0795 & 3.66 & 1.135 \\
$R 236 f a$ & -0.0907 & 3.3 & 1.12 \\
$S F_{6}$ & -0.1199 & 3.66 & 1.585 \\
$R 134 a$ & -0.1215 & 3.66 & 1.585 \\
Propane & -0.1305 & 3.66 & 1.585 \\
$R 152 a$ & -01366 & 3.66 & 1.585 \\
$C O_{2}$ & -0.1687 & 2.44 & 1.585 \\
\hline
\end{tabular}

The plots on the right of Figure 2 show a more typical behaviour, since most fluids do not exhibit a non-classical behaviour, and for those that do, the results discussed above show that the inlet stagnation conditions to the turbine would need to be highly supercritical $\left(p_{o} / p_{c} \approx 4\right)$. The plots on the right of Figure 2 show the variations in fundamental derivative and Prandtl-Meyer function for expansions across a turbine vane with sub-critical inlet stagnation conditions. In addition to $M D 4 M$, results for pentane and $S F_{6}$ are shown; pentane is a typical ORC working-fluid, and $S F_{6}$ is one of the fluids used for the experimental work discussed later. The results show that for sub-critical expansion at these conditions and close to the inversion curve, the fundamental derivative continuously rises as pressure drops, such that the nonclassical region is always avoided.

Figure 2 shows that for the sub-critical expansions shown here, the Prandtl-Meyer function continuously rises as the flow expands. The Prandtl-Meyer function is higher and also increases at a higher rate with Mach number for the dense gases shown here, as compared to air. This could play a significant role in trailing-edge flows where the flow meets an abrupt change in area, since large changes in PrandtlMeyer function with Mach number means that, conversely, the flow direction can be changed significantly with little change in flow Mach number.

Consider a generic supersonic flow around a trailingedge (Fig. 1), where we define the turning angle of the flow as it accelerates around the trailing-edge as $\theta_{w}$. In an inviscid flow of a gas with $\Gamma>1$, such as air, there is a maximum angle through which the flow can turn before a slip-stream 


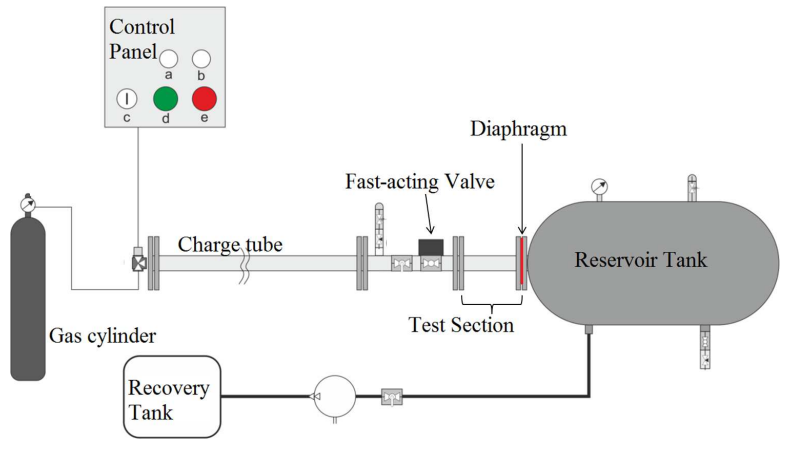

Fig. 3. SCHEMATIC OF LUDWIEG TUBE

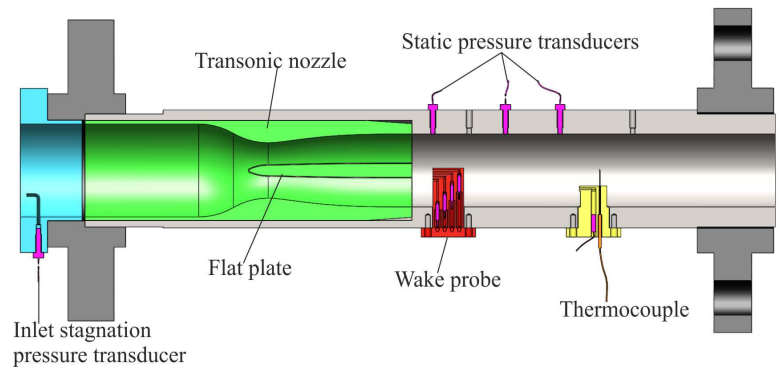

Fig. 4. SCHEMATIC OF THE TEST SECTION

is formed, and this is set by the asymptotic behaviour of the Prandtl-Meyer function as the Mach number approaches infinity. On the other hand, an inviscid flow of a gas with $\Gamma<1$ does not necessarily have such asymptotic behaviour [3], and indeed there may be no limit to the Prandtl-Meyer function as Mach number increases. In practise, the point of separation around the trailing-edge circle will be strongly dependent on viscous effects and therefore the value of $\theta_{w}$ will likely be a strong function of Reynolds number. Nonetheless, the behaviour of the Prandtl-Meyer function for dense gases suggests higher turning of the flow before separation may occur as compared to conventional gases such as air, steam or $\mathrm{CO}_{2}$.

\section{EXPERIMENT DESIGN AND SET-UP}

The results shown above (see Figure 2) show that in the range of Mach numbers $1<M<2$, pentane and $S F_{6}$ have similar values of fundamental derivative and $v$, provided they operate at the same reduced conditions. Therefore $S F_{6}$ was used in the experimental work as a model ORC working fluid, because it has similar gas dynamic behaviour to pentane which is a typical ORC fluid, but does not pose a health or fire risk. Furthermore experiments can be performed at ambient temperatures, since the critical temperature for $S F_{6}$ is roughly $39.1^{\circ} \mathrm{K}$ lower than that of pentane.

An experiment was designed where a supersonic trailing-edge flow was produced using a recently commissioned Ludwieg tube facility (Fig 3). The wake flow was produced by a plate embedded into an axisymmetric nozzle (Fig. 4) upstream of the working section. Three working fluids were tested (Air, $\mathrm{CO}_{2}$ and $\mathrm{SF}_{6}$ ). For each gas, a different nozzle was used in order to ensure tests were performed at

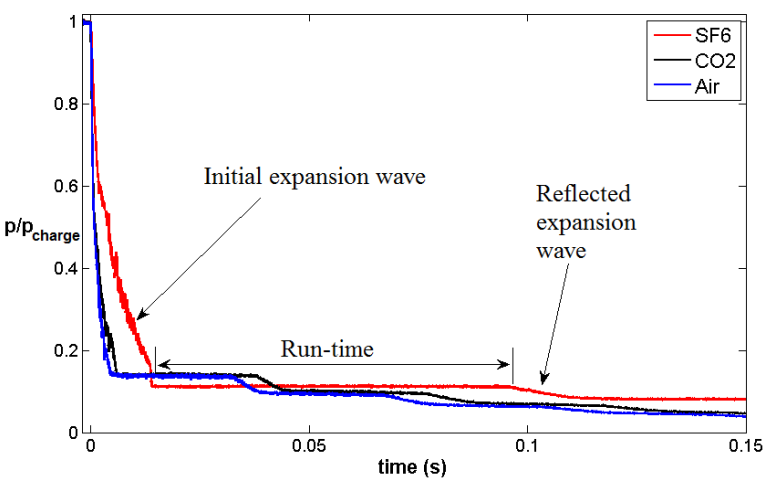

Fig. 5. PRESSURE RESPONSE FOR THE DIFFERENT RUNS NON-DIMENTIONALISED OVER THE CHARGE PRESSURE

the same Mach number. The nozzles were designed according to the method of characteristics suggested by Wheeler and Ong [3] which accounts for dense gas effects. The original $2 D$ nozzle design code of [3] was modified to produce axisymmetric nozzles with an exit Mach number of 2. Since the test Reynolds numbers were high $\left(\operatorname{Re}=o\left(10^{6}\right)\right)$, it was assumed that blockage effects from boundary layer growth could be neglected in the nozzle design; this was verified using CFD predictions of the nozzles to ensure that the design method achieved the desired conditions.

The rig is composed of a $5.7 \mathrm{~m}$ charge tube (diameter $50 \mathrm{~mm}$ ), a $0.4 \mathrm{~m}$ test section, a 150 litre reservoir tank, and a gas recovery system. The charge tube and test section are pressurised by small increments with the desired gas; the pressurisation is controlled with a fast acting valve. This ensured that the pressure and temperature field is homogeneous when the diaphragm bursts. The reservoir tank is evacuated to ensure a high pressure ratio. When using $\mathrm{SF}_{6}$ a "DILO B052R11 $\mathrm{SF}_{6}$-gas" service cart was used to ensure full recovery of the gas.

The gases chosen for the experiments were air, carbon dioxide $\left(\mathrm{CO}_{2}\right)$ and sulphur hexafluoride $\left(\mathrm{SF}_{6}\right)$. As discussed above, $\mathrm{SF}_{6}$ was selected because it has similar properties and behaviour to Pentane, which is a widely used gas in ORCs. Air and $\mathrm{CO}_{2}$ were chosen as validation and comparison real gases.

Figure 5 shows the measured wall static pressure within the test section for the three gases tested during a run. For all the gases, the general behaviour is as follows; immediately after the diaphragm burst an expansion wave propagates upstream (seen at $t=0 s$ ). Once the expansion wave passes upstream of the nozzle throat a supersonic flow is produced in the test section and constant run conditions are developed. The expansion wave travels up to the opposite end of the charge tube were it bounces back downstream. The supersonic flow in the test section is maintained until the reflected expansion wave re-enters the test section. In Figure 5 it can be seen that because $S F_{6}$ has an acoustic speed around three times slower than air the "run-time" of $S F_{6}$ is the longest one lasting $t_{\text {run }}=84.5 \mathrm{~ms}$, where $\mathrm{CO}_{2}$ and air are 28.8 and $33.2 \mathrm{~ms}$ long respectively.

The run conditions for each gas are shown in Tab. 2. The 
desired charge pressure was set by the bursting pressure of the diaphragm; the diaphragms were made from Mylar and a calibration of the diaphragm thickness required to achieve various burst pressures was performed in order to determine the diaphragm thickness required for each run. A heater jacket was used for the experiments using $\mathrm{CO}_{2}$; the heater jacket was used to pre-heat the $\mathrm{CO}_{2}$ to $120^{\circ} \mathrm{C}$, which was required in order to avoid two-phase flows during the test-run. The Reynolds number range for the different test conditions are from 0.9 to 1.5 Million based on the trailing-edge thickness, $t_{\text {t.e. }}$, and this is shown later to have little effect on the trailing-edge flow field. It should be noted that while air and $\mathrm{CO}_{2}$ have values of the Fundamental derivative greater than one throughout the expansions, the Fundamental derivative of $S F_{6}$ achieves values lower than one, and this represents a gas behaviour within the dense gas region. However, the gas is not expected to enter the non-classical region.

Table 2. EXPERIMENTAL RUN CONDITIONS FOR EACH GAS

\begin{tabular}{|l|c|c|c|c|c|}
\cline { 2 - 6 } \multicolumn{1}{c|}{} & $\mathbf{p} / \mathbf{p}_{\mathbf{c}}$ & $\mathbf{T} / \mathbf{T}_{\mathbf{c}}$ & $\mathbf{R e}$ & $\mathbf{M}$ & $\Gamma$ \\
\hline Air & 0.29 & 2.37 & $0.95 \times 10^{6}$ & 1.99 & 1.2 \\
\hline $\mathbf{C O}_{2}$ & 0.14 & 1.11 & $0.90 \times 10^{6}$ & 1.97 & $1.13-1.15$ \\
\hline $\mathbf{S F}_{6}$ & 0.32 & 0.95 & $1.44 \times 10^{6}$ & 2.04 & $0.99-1.04$ \\
\hline
\end{tabular}

To capture wake measurements a stagnation pressure probe with four "Kulite XCL-62" pressure transducers was placed 1.5 trailing-edge thicknesses downstream of the plate trailing-edge aligned with the centre of the plate/test-section (Fig 4). The probe was produced using laser sintering 3Dprinting. This allowed for the design to be compact and easily manufactured, hence various designs were tested to determine minimum blockage. The final design was a wedge probe configuration, with a wedge angle of $10^{\circ} \mathrm{deg}$, which widens slightly at the base to accommodate the sensors inside. The sensors were mounted within the probe to ensure response times were minimized, but this limited the number of sensors to a maximum of four sensors. In order to get more measurements within the wake and a greater point density, runs were performed with the probe set at several distances from the centre of the test section.

Simultaneously, flush mounted wall static pressure sensors and an inlet stagnation pressure probe using "Kulite XTEL-140" transducers were used to measure the expansion of the flow and calculate Mach numbers in the test Section. Pressures were taken using fast-response transducers which were sampled at $100 \mathrm{KHz}$ using the National instruments" "USB-6356 X-series DAQ" data acquisition system. All pressure probes were calibrated using the high precision modular pressure controller GE PACE5000. The pre-run gas temperature was recorded using a fast response needletype "OMEGA Model HYP0" probe. The uncertainty in the measured pressure was estimated to be $600 \mathrm{~Pa}$ (which corresponds to $\approx 0.6 \%$ uncertainty in measured pressure ratio)

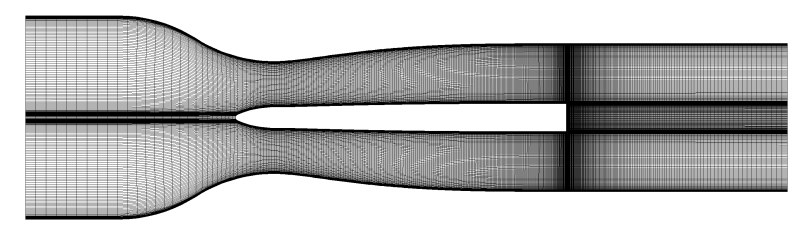

Fig. 6. NOZZLE WITH PLATE MESH

and the temperature measurement sensitivity was $0.6 K$. Run to run differences in test condition pressure ratio was determined to be $<0.2 \%$.

\section{COMPUTATIONAL SET-UP}

Two CFD studies were designed; the first is a CFD simulation of experimental geometry where results are analysed and compared to the experiments. The aim of this is to validate numerical models for dense gas wake flows by comparing results with the experiments. The second is a turbine vane flow, where the same methods used for the flat-plate are used to investigate the flow within a typical ORC turbine vane passage.

The flow solver software used was FLUENT v14.5, where all simulations were calculated using the density based solver. Steady simulations were performed using the second order implicit algorithm. Unsteady simulations were also performed using a dual-time-stepping method.

For the flat-plate study, the boundary conditions set were the inlet total pressure and temperature and the exit static pressure, which were determined from the experimental data. RANS modelling was performed using a vorticity based Spalart-Allmaras (SA) turbulence model. Other RANS models $(\kappa-\omega, \kappa-\varepsilon$, Reynolds Stress) were studied, however they all tended to give results which differed significantly from the experimental data (for reasons discussed later) and so only the SA model, which showed the closest agreement between CFD and experiment, is discussed here. The value of $y^{+}$at the first grid point away from the wall was typically $y^{+} \gtrsim 30$, so that wall functions were employed by the solver.

For the study of the flat-plate wake, a model was produced which included the transonic nozzle, the plate and the working section downstream. This model was imported into the software package of ANSYS, ICEM CFD 14.5, which was used to create structured meshes of the computational domain. Figure 6 shows an example of the mesh on a plane perpendicular to the plate, at the centre of the working section. A mesh sensitivity analysis was performed; solutions using three meshes of $0.7,2$ and 4 million points were compared, where the $4 \mathrm{M}$ mesh was preferred for accuracy. $4 \mathrm{M}$ simulations for $\mathrm{CO}_{2}$ could not be achieved due to stability issues. The resulting difference in loss coefficient at the exit between the 2 million points mesh and the 4 million mesh was $2 \%$. Therefore, it was concluded that the $2 \mathrm{M}$ mesh would give an acceptable accuracy.

Large Eddy Simulations (LES) were also performed. For this the Smagorinsky-Lilly model was employed, where $C_{s}=0.1$ and $P r_{\text {wall }}=0.85$. Assuming the characteristic 


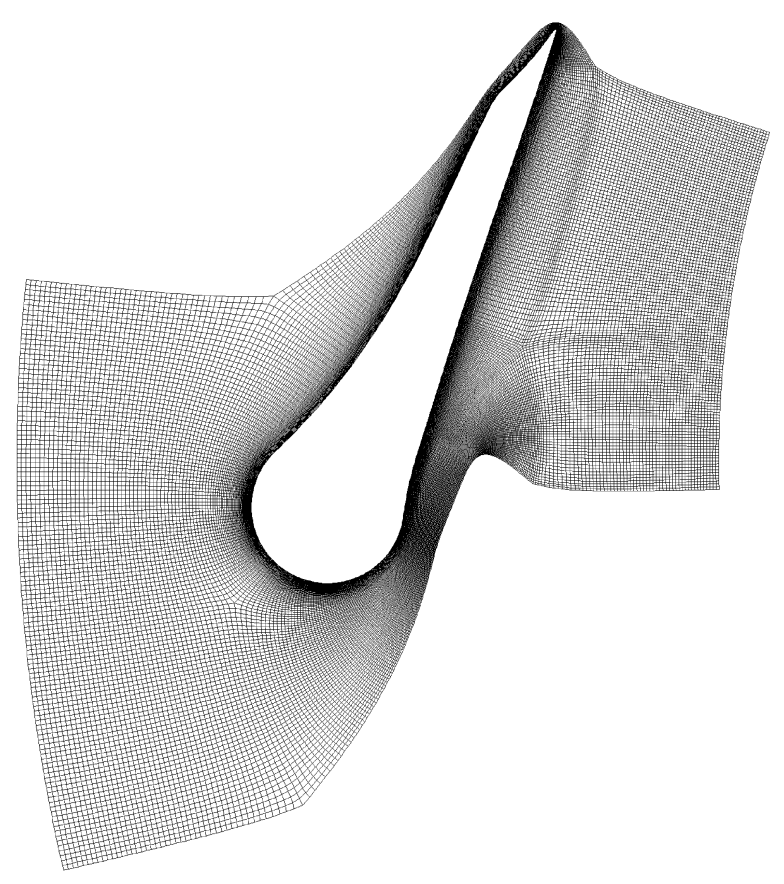

Fig. 7. TURBINE VANE MESH

length-scale of the unsteady flow was roughly equal to the trailing-edge thickness, the largest time-scale was estimated from the freestream velocity and trailing-edge thickness, the chosen time-step was then set to be equal to 10 times smaller than this value.

The second CFD study was a typical turbine vane flow with pentane as a working fluid. The model was designed in a previous study [19] and is representative of an ORC transonic turbine. The turbine stage was designed to have operating conditions of a rotational speed of 26500RPM, mass flow rate of $2 \mathrm{~kg} / \mathrm{s}$, power output of $155 \mathrm{~kW}$, a temperature ratio $T_{\circ, \text { in }} / T_{\text {exit }}=1.15$ and a pressure ratio $p_{\circ, \text { in }} / p_{\text {exit }}=11$ across the turbine. The inlet stagnation reduced conditions were $p_{\circ, \text { in }} / p_{c}=0.59$ and $T_{\circ, \text { in }} / T_{c}=0.96$. The resulting range of fundamental derivative values is $0.75<\Gamma<0.95$ across the vane.

The vane was produced using a Matlab script to produce the quasi-3D geometries and ANSYS turbogrid v14.5 for the meshing (Fig. 7). The meshing of the vane geometries (Fig. 7) was performed using similar mesh resolution for the flatplate flat plate study as this is the mesh that has been validated to give acceptable results. Hence the wall $y^{+}$along the surface of the vane has been set so that it is generally $y^{+} \gtrsim 30$ and wall functions are used, and a similar mesh density has been produced at the trailing-edge, with 50 points along the trailing-edge height $\left(t_{\text {t.e. }}=0.5 \% c\right)$ and 40 points along the $\operatorname{span}(\operatorname{span}=5 \% c)$. As a result wall functions are used everywhere apart from the base separation region.

In all simulations air was modelled as an ideal gas, where $\mathrm{CO}_{2}, \mathrm{SF}_{6}$ and pentane flows were modelled as real gases using the NIST thermodynamic models in FLUENT.

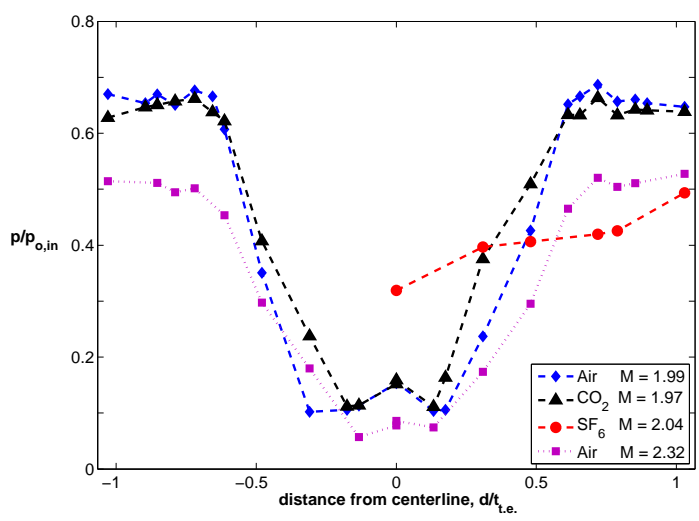

Fig. 8. TOTAL PRESSURE MEASUREMENTS IN WAKE OF SEVERAL GASES AT $1.5 t_{\text {t.e. }}$ FROM TRAILING-EDGE

\section{EXPERIMENTAL RESULTS}

Figure 8 shows the total pressure measured by the wake wedge probe. Wherever the flow is supersonic the probe produces a bow shock ahead of it, hence the measured values of total pressure will be lower than the actual total pressure in regions of supersonic flow due to the total pressure loss that exists across a normal shock wave. This shock loss will be highest in the free-stream where the Mach numbers are highest and will reduce within the wake, being zero wherever the flow is subsonic.

Four different runs were performed: three runs at the same Mach number $M=2$ using air, $\mathrm{CO}_{2}$ and $\mathrm{SF}_{6}$; and a fourth run for air at a higher Mach number of $M=2.32$. The total pressure profiles for air and $\mathrm{CO}_{2}$ start at around $p_{\circ} / p_{\text {in }}=0.65$ and show a large fall in pressure within the wake, which has a width similar to the trailing-edge thickness of $t_{\text {t.e. }}=7.5 \mathrm{~mm}$. The centre of the wakes have a relatively flat variation in total pressure at around $p_{\circ} / p_{0}$,in $=0.1$ over a distance of around $53 \%$ of the trailing-edge thickness for air, and $40 \%$ for $\mathrm{CO}_{2}$. On the other hand, the measured profile for $S F_{6}$ has a lower measured total pressure in the free-stream (around $p_{\circ} / p_{\text {o,in }}=0.5$ ) but a higher total pressure within the wake, which has a minimum value of $p_{\circ} / p_{\text {o,in }}=0.3$. It should be noted that for the case of $S F_{6}$ due to the complexities of testing with this gas it was only possible to obtain measurements over half of the wake, but the measurements with other gases confirmed the symmetry of the flow in the working section.

A further experiment was performed to assess the sensitivity of the air wake profile to Mach number and this data is also shown in Fig. 8. The data shows that the increase in Mach number from 1.99 to 2.32 produces a lower freestream measured total pressure as expected because of the increased shock loss at the higher Mach number. Nonetheless, the shape of the wake remains relatively unchanged with a slight reduction in total pressure at the centre of the wake.

There is a radical change in wake shape at the dense gas conditions $(\Gamma<1)$ tested here which cannot be attributed to Mach number, or indeed Reynolds number (as will be shown later), but instead is a result of the change in the gas dynamics at the trailing-edge, as discussed next. 


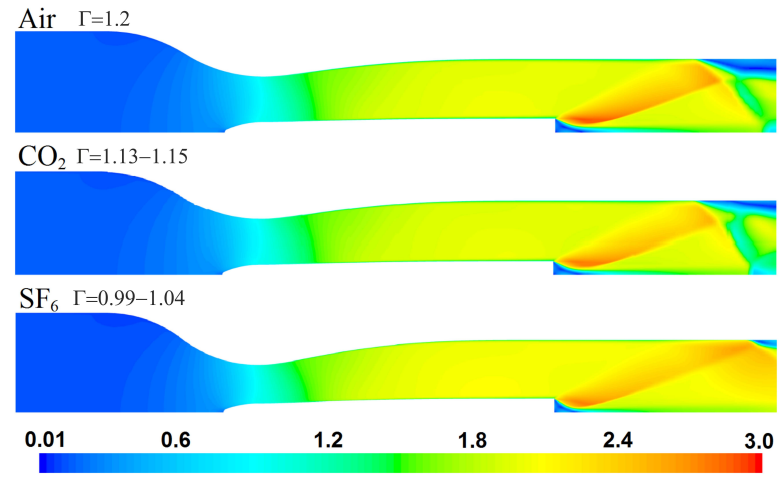

Fig. 9. PREDICTED MACH CONTOURS FROM RANS SIMULATIONS AT RE $\approx 1.5 \mathrm{M}$ and $\mathrm{M} \approx 2.0$

\section{COMPUTATIONAL RESULTS}

\section{Supersonic wake simulations}

In this section we discuss the CFD simulations of the experimental set-up, and compare the experimental data to the CFD in order to validate the computational method.

Figure 9 shows the predicted Mach number contours on a plane perpendicular to the plate, at the centre of the working section from RANS simulations of the experimental test runs. Here it can be seen that in all cases, the flow is continuously accelerated through the nozzle up to the trailing-edge plane, reaching a Mach number of 2 at the trailing-edge. At the trailing-edge, the flow is accelerated further through a Prandtl-Meyer expansion fan, as the flow is turned around the trailing-edge corner. Once the flow separates, a free shear layer develops downstream of the trailing-edge. The concave curvature of the shear layer leads to the development of compression waves which coalesce to form an oblique shock. There then forms a wake flow downstream of this shock.

Figure 9 shows that as the fundamental derivative decreases, the region of separated flow within the base region of the trailing-edge reduces in size, demonstrating that the flow is turned through an increasingly higher angle as $\Gamma$ reduces. There is also a reduction in the Mach number ahead of the oblique shock as $\Gamma$ reduces, and this also demonstrates the reduction in sensitivity of the Mach number to the change in area downstream of the trailing-edge when $\Gamma$ is reduced. Both of these effects are a consequence of the variation in Prandtl-Meyer function as $\Gamma$ reduces, which was discussed previously. As expected, the gas presents dense gas behaviour only and the non-classical region is not reached.

In Figure 10, total pressure values from the CFD are compared to the experimentally measured total pressure within the wake; for these cases the total pressures from the CFD calculations have been converted to account for the shock loss which occurs across the bow wave of the total pressure probe in region of supersonic flow, so that a correct comparison between the CFD and experiment can be made; this total pressure will be referred to as the shocked total pressure. This was performed by using the REFPROP real gas routines to determine the total pressure loss based on the CFD predicted Mach number and gas properties at the measurement plane. The figure shows that there are significant

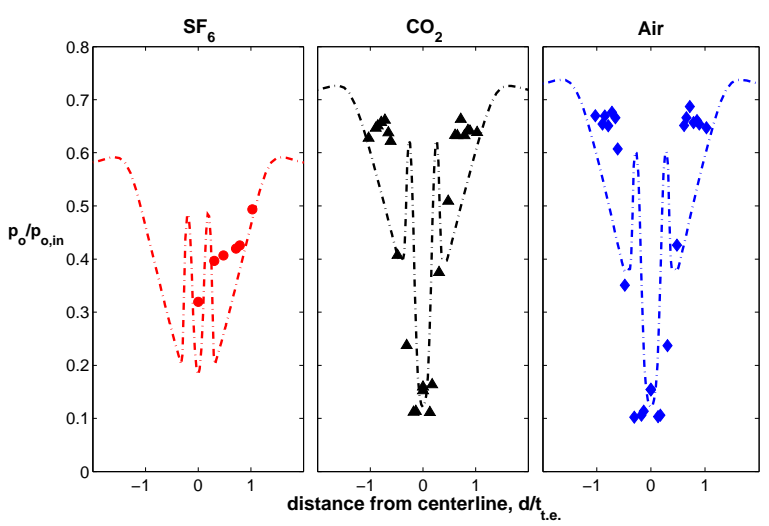

Fig. 10. COMPARISON OF SHOCKED TOTAL PRESSURE FROM EXPERIMENTAL DATA AND RANS SIMULATIONS AT $1.5 t_{\text {t.e. }}$ FROM TRAILING-EDGE

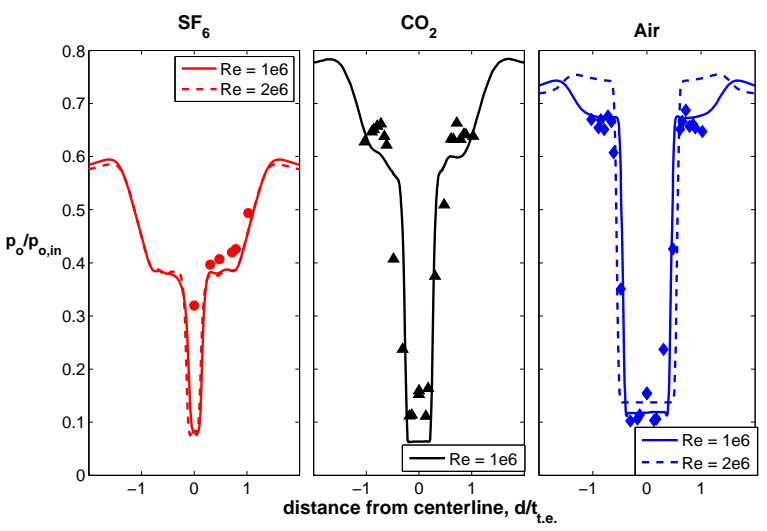

Fig. 11. COMPARISON OF SHOCKED TOTAL PRESSURE FROM EXPERIMENTAL DATA AND LES SIMULATIONS AT 1.5 t.e. FROM TRAILING-EDGE

differences between measured and computed wakes: Firstly, the RANS simulations seems to over predict the Mach number at the edges of the wake, causing a lower shocked total pressure; within the wake, the region of subsonic flow also appears to be limited to a much thinner region within the wake, as compared to the experiment. These differences are suggestive of a much higher degree of pressure recovery occurring within the CFD as compared to the experiment, which indicates that the RANS modelling is over-predicting the generation of eddy viscosity in this region. This is unsurprising, since the turbulence within the free-shear is unlikely to be in an equilibrium state.

In an attempt to improve the prediction of the flow, Large Eddy Simulations (LES) were performed using a Smagorinsky-Lilly model (as discussed earlier). Figure 11 shows the predicted shocked total pressure with the experimental data from LES simulations with Air, $\mathrm{CO}_{2}$ and $\mathrm{SF}_{6}$ as working fluids. The agreement is very encouraging; the LES predictions capture the large change in wake profile observed in the experiment between Air, $\mathrm{CO}_{2}$ and $\mathrm{SF}_{6}$. The results in this figure show results from simulations performed at two different Reynolds numbers of 1 and 2 million, to cover the 


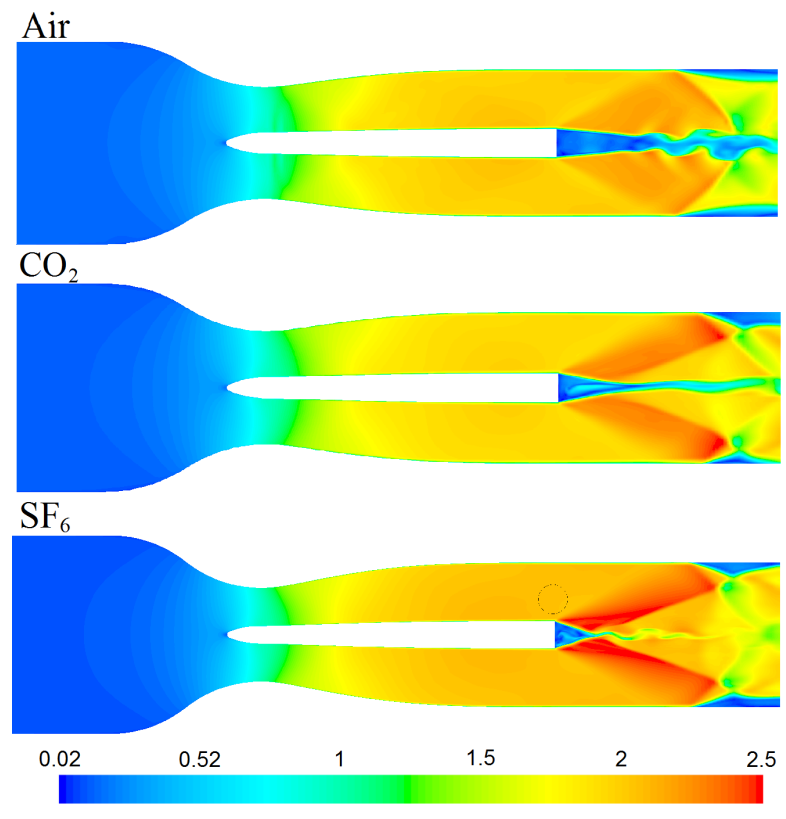

Fig. 12. LES PREDICTED MACH NUMBER CONTOURS OF (FROM TOP TO BOTTOM) AIR, $\mathrm{CO}_{2}$ AND SF 6 WAKES AT RE $\approx$ $1.5 \mathrm{M}$ and $\mathrm{M} \approx 2.0$

range of Reynolds numbers in the experiment. The sensitivity of the wake to Reynolds number is most apparent for the Air simulations, while the $S F_{6}$ simulations are relatively insensitive to the difference in Reynolds number. The results show that the effect of this large change in wake shape is largely independent of Reynolds number, and is therefore a strong function of $\Gamma$.

Figure 12 shows predicted contours of Mach number from the LES for Air, $\mathrm{CO}_{2}$ and $S F_{6}$. For air, the flow presents very low separation angles, $\theta_{w}$, as it passes the trailingedge, producing very weak shocks and expansions throughout. This means that the wake boundary is almost parallel to the plate surface and results in a very wide wake. On the contrary, for $\mathrm{CO}_{2}$ and $\mathrm{SF}_{6}$, the flow experiences increasingly higher separation angles at the trailing-edge, which result in an over-expansion of the flow produced by a strong expansion fan at the trailing-edge and a closed region of separation behind the trailing-edge. The separation region is followed by strong compressions at either side which develop into oblique shock-waves, which turn the flow back into the stream-wise direction. The resultant wake from the closed region of separation is very thin.

Losses are determined by the increase of entropy across a domain and can be typically estimated using the base pressure in wake flows [20]. In Figure 13 the predicted values of base pressure, $p_{b}$, and mass-weighted average loss coefficient, $\zeta$, are plotted. Here the loss coefficients are computed using the local entropy values:

$$
\zeta=\frac{T_{s} \Delta s}{h_{o, \text { in }}-h_{s}}
$$
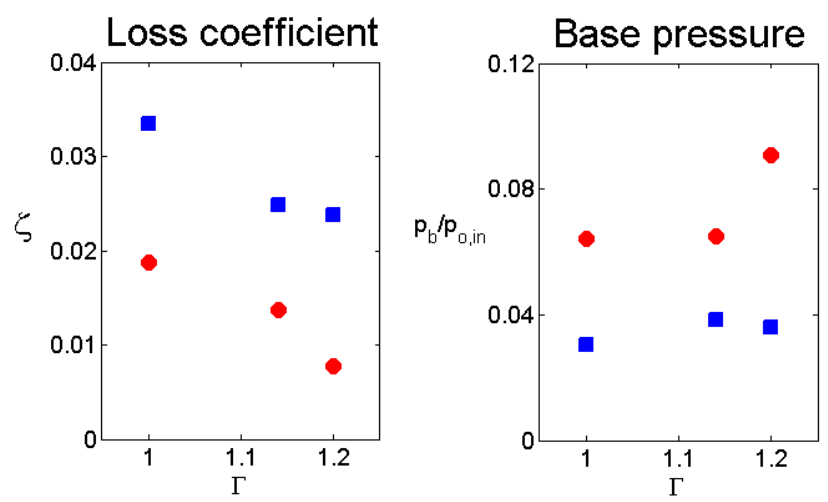

Fig. 13. RANS (घ) AND LES $(\bullet)$ COMPARISON OF MASSWEIGHTED AVERAGED LOSS COEFFICIENT AT $1.5 t_{\text {t.e. }}$ FROM TRAILING-EDGE AND BASE PRESSURE
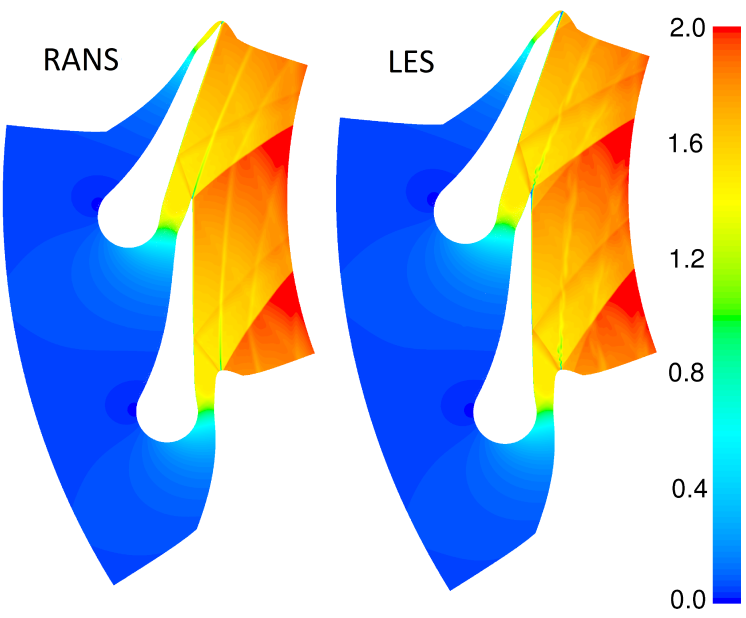

Fig. 14. PREDICTED MACH NUMBER CONTOURS OF PENTANE THROUGH RADIAL VANES

Where $T_{s}$ and $h_{s}$ are the local mass-weighted averages of isentropic enthalpy and temperature. The mass averaged loss coefficient has been calculated at a plane $1.5 t_{\text {t.e. }}$ downstream of the trailing-edge. Both the RANS and LES results show similar trends in loss coefficient and base pressure for all gas flows. The results show that a reduction in fundamental derivative leads to an increase in loss, with LES predicting lower losses. On the other hand, base pressure decreases with a reduction in fundamental derivative, with LES predicting higher pressures. Base pressure is very sensitive to the size of the separation region, with smaller separation regions resulting in lower base pressures. Comparing Fig 13 to Fig 12 it can be seen that most of the losses in the trailing-edge area come from inviscid losses related to the trailing-edge separation angle. Hence as the wake width decreases with larger separation angles, caused by a reduction in $\Gamma$, the losses are increased.

\section{Turbine vane simulations}

In this section we discuss the CFD simulations of the ORC turbine vane using both RANS and LES methods, sim- 


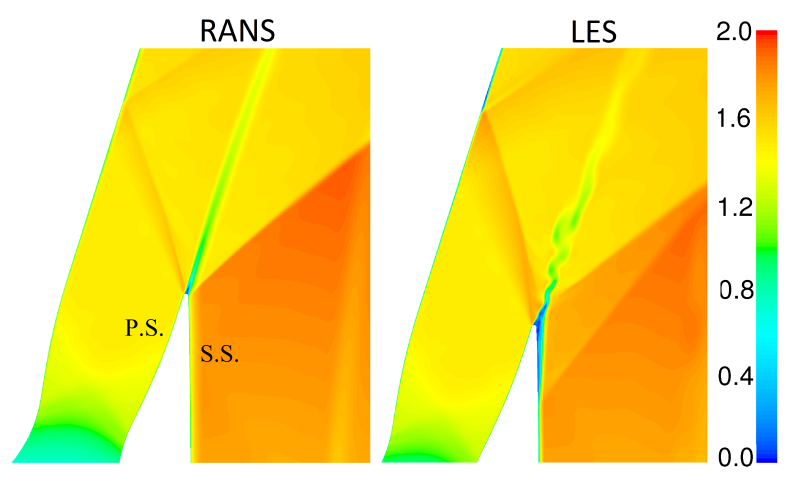

Fig. 15. PREDICTED MACH NUMBER CONTOURS OF PENTANE AT THE TRAILING EDGE
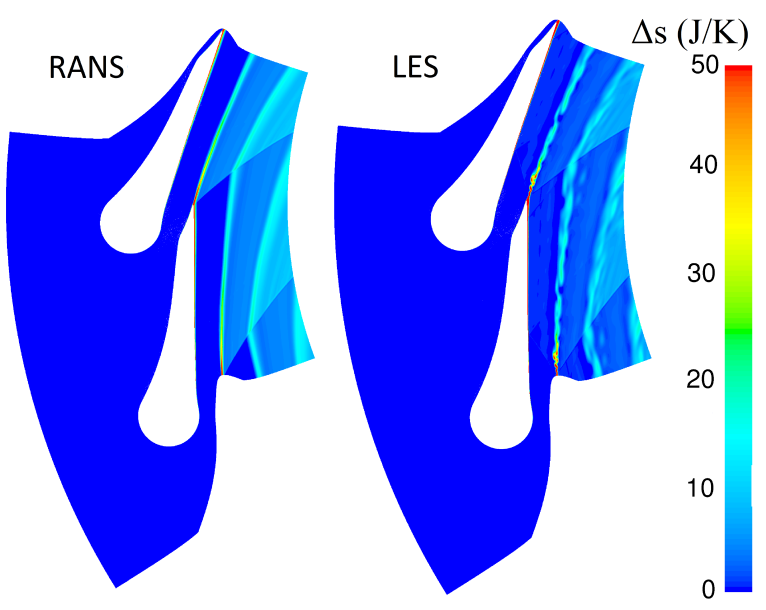

Fig. 16. ENTROPY CONTOURS OF PENTANE

ilarly to the flat plate study. Figure 14 shows Mach number contours of pentane calculated for both RANS and LES simulations. The flow enters the vane at a subsonic speed and is accelerated up to a supersonic Mach number at the trailingedge; on the suction-side (S.S.) the trailing-edge Mach number is $M \approx 2.1$, while on the pressure-side (P.S.) the trailingedge Mach number is around 1.3. The shock pattern downstream of the trailing-edge is slightly different for the two modelling approaches.

Figure 15 shows in more detail the flow structure at the trailing-edge. Similarly to flat plate results, in the case of the RANS, a strong oblique shock is formed on the suction-side (S.S.) ahead of the trailing-edge, and the base separation region is relatively small. In contrast, the LES predicts a flow separation before the trailing-edge, leading to a large separation zone in the region of the trailing-edge. This separation produces a weak compression, which reduces the Mach number at the trailing-edge and also reducing the subsequent trailing-edge shock strength. Likewise, this produces slightly stronger expansion fan and shock wave on the pressure-side (P.S.) of the trailing-edge.

The results from the vane simulations are consistent with previous results seen for the wake study above. Similarly to the flat-plate model, a larger separation is seen in the LES results as compared to the RANS simulation (Figs. 14 and

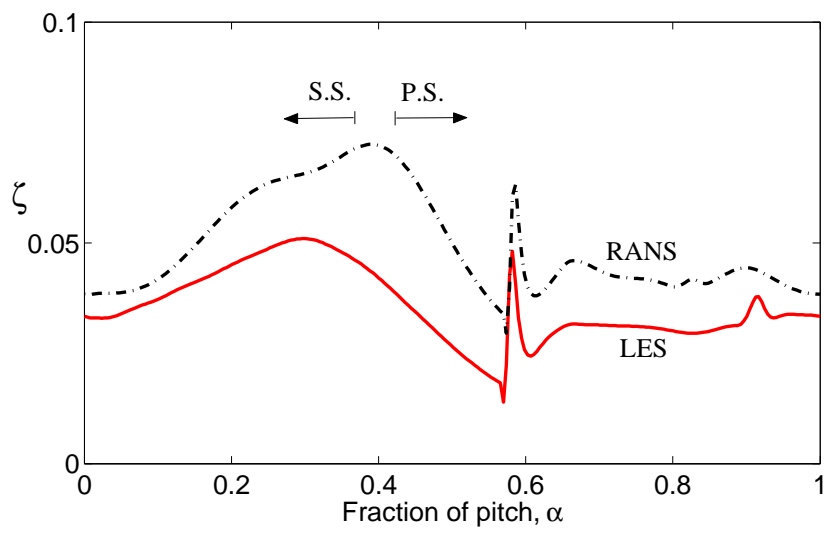

Fig. 17. LOSS COEFFICIENT DISTRIBUTION AT EXIT PLANE OF TURBINE VANE

15), which results in a deeper wake. Interestingly, the increased viscous loss is likely to result in a reduction in shock loss, since the increased base region seen in the LES predictions tends to reduce the pressure side trailing-edge shock strength. This is confirmed by the predicted entropy shown in Fig. 16, which shows a much higher entropy rise across the trailing-edge shock for the RANS case, as compared to the LES prediction.

Figure 17 shows the variation of loss at the exit of the computational domain for both the LES and RANS predictions. This clearly shows an increased loss for the RANS as compared to the LES, due to the increased shock loss. The position of the trailing-edge shock at the outlet for both predictions is at $\alpha \approx 0.58$, where $\alpha$ is the fraction of pitch. The mixing of the flow downstream of the trailing-edge makes it hard to separate viscous from inviscid losses, but it is estimated that the wake occupies the range of fraction of the pitch from $\alpha \approx 0.1$ to $\alpha \approx 0.57$ for both simulations. Table 3 shows the total loss coefficient and the contribution of the wake and freestream to the total loss for both LES and RANS cases, determined from the mass-weighted average of entropy within the wake and freestream. Both the RANS and LES simulations predict contributions from the wake to be significantly larger than contributions from the freestream (almost two-thirds of the total loss). The vane exit angle predicted by both the RANS and LES was $70.3^{\circ}$. Overall, the LES simulations predict a lower loss coefficient than the RANS, and this is partly due to a reduction in shock-loss.

Table 3. VANE LOSS COEFFICIENT AT EXIT PLANE

\begin{tabular}{|l|c|c|c|}
\cline { 2 - 4 } \multicolumn{1}{c|}{} & $\zeta$ & $\%$ from wake & $\%$ from freestream \\
\hline RANS & 0.047 & $62.2 \%$ & $37.8 \%$ \\
\hline LES & 0.035 & $61.6 \%$ & $38.4 \%$ \\
\hline
\end{tabular}




\section{CONCLUSIONS}

This paper described an investigation of the influence of dense gas effects on trailing-edge flows in a transonic ORC turbine. Exploration of the gas properties and Prandtl-Meyer function showed that dense gas effects could potentially increase supersonic turning of the flow at a trailing-edge. Experiments were performed of supersonic wake flows using a range of working fluids at similar Mach and Reynolds numbers. The data was used to validate computational simulations using both RANS and LES. The results showed RANS predictions failed to capture the correct trailing-edge flow structure. Instead, LES modelling gave a much closer agreement to the experimental data. This was thought to be due to an over production of eddy viscosity in the base region.

The results showed that within the dense gas region, the size of the base flow separation downstream of the trailingedge was significantly reduced as compared to gases which do not exhibit dense-gas effects. This is consistent with the increase in the Prandtl-Meyer function and thus supersonic turning achievable in the dense gas region. The results suggest that the viscous contributions to trailing-edge loss are reduced with working fluids operating within a dense-gas region, while inviscid contributions are significantly increased. This was shown to be largely independent on Reynolds number, for the range of conditions tested here.

An LES simulation of an ORC turbine vane with an exit Mach number of 1.35 and operating with Pentane was also performed, and this showed that about two-thirds of the total loss was due to the viscous effects, and the remaining third was a result of shocks generated within the trailing-edge region. Viscous effects could be further improved by better design of the suction surface of the vane near the trailingedge.

The results indicate that the balance between viscous and shock loss is a strong function of fundamental derivative, which will be largely dependent on the choice of working fluid and turbine conditions.

\section{Acknowledgements}

This work was supported by the EPSRC (EP/J006394/1 and EP/L027437/1) and GE Global Research.

The authors would like to thank Nalleli Góngora-Orozco for her contribution in designing and building the experimental rig and set-up.

\section{References}

[1] Thompson, P., 1971. "Fundamental derivative in gas dynamics". Physics of Fluids, 14(9), pp. 1843-.

[2] Lambrakis, K., and Thompson, P., 1971. "Theoretical study of negative shock waves in real fluids". Bulletin of the American Physical Society, 16(11), p. 1320.

[3] Wheeler, A., and Ong, J., 2013. "The role of dense gas dynamics on orc turbine performance". In ASME Turbo Expo, San Antonio, Texas

[4] Cramer, M., and Crickenberger, A., 1992. "Prandtl- meyer function for dense gases". AIAA Journal, 30(2), pp. 561-564.

[5] Borisov, A., Borisov, A., Kutateladze, S., and Nakoryakov, V., 1983. "Rarefaction shock-wave near the critical liquid vapor point". Journal of Fluid Mechanics, 126, January, pp. 59-73.

[6] Lambrakis, K., and Thompson, P., 1972. "Existence of real fluids with a negative fundamental derivative gamma". Physics of Fluids, 15(3), p. 933.

[7] Thompson, P., and Lambrakis, K., 1973. "Negative shock-waves". Journal of Fluid Mechanics, 60, August, pp. 187-208.

[8] Cramer, M., 1989. "Negative nonlinearity in selected fluorocarbons". Physics of Fluids, 1(11), pp. 18941897.

[9] Aldo, A., and Argrow, B., 1995. "Dense gasflow in minimum length nozzles". Journal of Fluids Engineering-Transactions of the ASME, 117(2), pp. 270-276.

[10] Brown, B., and Argrow, B., 1997. "Two-dimensional shock tube flow for dense gases". Journal of Fluid Mechanics, 349, October, pp. 95-115.

[11] Monaco, J., Cramer, M., and Watson, L., 1997. "Supersonic flows of dense gases in cascade configurations". Journal of Fluid Mechanics, 330, pp. 31-59.

[12] Cramer, M., and Crickenberger, A., 1991. "The dissipative structure of shock-waves in dense gases". Journal of Fluid Mechanics, 223, February, pp. 325-335.

[13] Congedo, P., Corre, C., and Cinnella, P., 2011. "Numerical investigation of dense-gas effects in turbomachinery”. Computers \& Fluids, 49, pp. 290-301.

[14] Anderson, W., 1991. "Numerical study on using sulfurhexaflouride as a wind-tunnel test gas". AIAA Journal, 29(12), pp. 2179-2180.

[15] Anders, J., Anderson, W., and Murthy, A., 1999. "Transonic similarity theory applied to a supercritical airfoil in heavy gas". Journal of Aircraft, 36(6), pp. 957-964.

[16] Kluwick, A., 2000. "Marginally separated flows in dilute and dense gases". Philosophical Transactions of the Royal Society of London Series A - Mathematical Physical and Engineering Sciences, 358, December, pp. 3169-3192.

[17] Kluwick, A., 2004. "Internal flows of dense gases". Acta mechanica, 169, May.

[18] Cinnella, P., and Congedo, P., 2007. "Inviscid and viscous aerodynamics of dense gases". Journal of Fluid Mechanics, 580, pp. 179-217.

[19] Wheeler, A., and Ong, J., 2014. "A study of the threedimensional unsteady real-gas flows within a transonic orc turbine". In ASME Turbo Expo, Dusseldrof, Germany

[20] Denton, J. D., and Xu, L., 1990. "The trailing edge loss of transonic turbine blades". Journal of Turbomachinery, 112, April, pp. 277-285.

[21] Lemmon, E., and Span, R., 2006. "Short fundamental equations of state for 20 industrial fluids". Journal of Chemical Engineering Data, 51, pp. 785-850.

[22] Lemmon, E., Huber, M., and McLinden, M., 2010. 
NIST Standard Reference Database 23: Reference Fluid Thermodynamic and Transport PropertiesREFPROP, Version 9.0. National Institute of Standards and Technology, Standard Reference Program, Gaithesburg.

[23] Span, R., and Wagner, W., 2003. "Equations of state for technical applications. ii. results for nonpolar fluids". Journal of Fluid Mechanics, 580, p. 179. 